\section{Epidemiología del sobrepeso y obesidad infanto-juvenil en las comunas de Concepción, Coronel y Hualpén, VIII Región de Chile}

\author{
NATALIA ULLOA ${ }^{1, a}$, JORGE SAPUNAR ${ }^{2}$, PAULA BUSTOS ${ }^{1, b}$, KATIA SÁEZ ${ }^{3, c}$, \\ SYLVIA ASENJO ${ }^{4}$, MARCELA TAIBO $^{5, \mathrm{a}}$, AMALIA CORNEJO $^{5, \mathrm{~d}}$
}

\section{Frequency of obesity and overweight among school age children living in southern Chile}

Background: The frequency of obesity is increasing steadily in Chile. Aim: To assess the prevalence of obesity and overweight in children and teenagers living in three southern Chilean cities. Material and Methods: The database of an evaluation performed in 2006 in schools, was used to obtain weight and height of 32514 subjects aged $12 \pm 4$ years (48\% males). Criteria proposed by the International Obesity Task Force (IOTF) and the Centers for Disease Control (CDC) were used to define obesity and overweight. Results: According to CDC criteria the prevalence of overweight and obesity was $11.2 \%$ and $6.5 \%$, respectively. According to IOTF criteria, the figures were 13.2 and 4\%, respectively. The higher frequency of overweight and obesity was observed among children aged less than eight years. Conclusions: There is a high frequency of obesity and overweight in the studied sample.

(Rev Med Chile 2010; 138: 1365-1372).

Key words: Childhood; Obesity; Overweight.

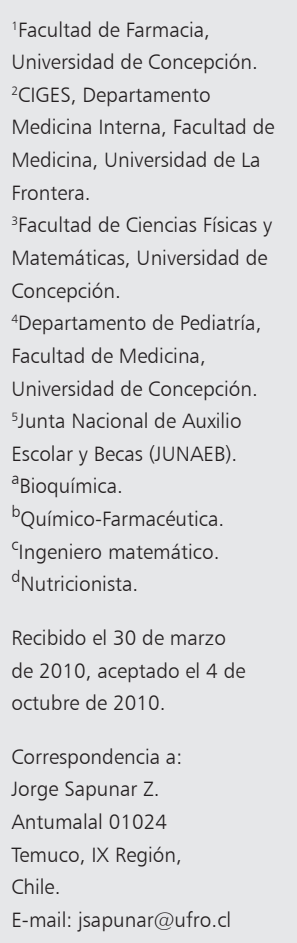

$\mathrm{E}$ 1 sobrepeso y obesidad afectan a una importante proporción de la población adulta en nuestro país ${ }^{1}$, constituyendo un problema de salud pública relevante por el exceso de morbilidad y mortalidad asociado a estos trastornos nutricionales.

La importancia del sobrepeso y obesidad entre escolares y adolescentes ha sido estudiada en diversas naciones del mundo. En todos los casos, independientemente del sexo y grupo etario considerado, se aprecia un aumento en la prevalencia del sobrepeso y la obesidad infanto-juvenil al comparar cortes transversales sucesivos ${ }^{2-22}$.

En nuestro país Kain y colaboradores ${ }^{23}$ estudiaron la prevalencia del sobrepeso y obesidad en escolares chilenos que ingresaban a $1^{\circ}$ básico entre los años 1987 y 2000, encontrando tasas de prevalencia semejantes a la de países como España.
Global School-Based Student Health Survey (GSHS) ${ }^{24}$ fue un estudio multicéntrico internacional que incluyó establecimientos educacionales de nuestro país y que fue patrocinado por International Obesity Taskforce (IOTF) y Centers for Disease Control and Prevention (CDC). Su propósito fue obtener datos de conductas en salud y factores protectores entre los estudiantes de $7^{\circ}$ básico a $1^{\circ}$ medio. En nuestro país los investigadores encontraron que 3,7\% de los escolares encuestados tenía obesidad y $20 \%$ sobrepeso.

El estudio de Kain sólo consideró niños de $1^{\circ}$ básico y GSHS obtuvo los datos para calcular el índice de masa corporal (IMC) mediante una encuesta. El utilizar el auto-reporte de peso y talla subestima significativamente la prevalencia de sobrepeso y obesidad en relación a la antropometría ${ }^{25}$. Por lo anterior nos pareció necesario 
obtener nuevos datos para conocer la importancia de los trastornos nutricionales infanto-juveniles en nuestro país.

El propósito de nuestra investigación fue conocer la prevalencia de sobrepeso y obesidad infanto juvenil en las comunas de Concepción, Coronel y Hualpén. Adicionalmente buscamos identificar grupos de riesgo para estos trastornos nutricionales en la muestra de población de interés.

La información obtenida en este estudio se utilizó para la implementación del Proyecto MECESUP UCO 0609 "Modelo Educativo Transdisciplinario para la Prevención de la Obesidad Infanto-Juvenil" de la Facultad de Farmacia de la Universidad de Concepción.

\section{Material y Métodos}

Se solicitó a la Junta Nacional de Auxilio Escolar y Becas (JUNAEB) los resultados de la evaluación realizada a los establecimientos educacionales bajo su supervisión el año 2006 en las comunas de Concepción, Coronel y Hualpén de la Región del Bío-Bío. La base de datos aportada por la JUNAEB contenía información de 49.199 alumnos para las variables fecha de nacimiento (día, mes y año), nombres y apellidos, género, peso (kg) y talla $(\mathrm{cm})$, escolaridad de la madre y del jefe de hogar, índice de prioridad social familiar ${ }^{26}$, ubicación del establecimiento educacional y tipo de enseñanza impartida.

Se incluyeron sólo los alumnos entre 6 y 18 años con datos de antropometría completos (peso y estura), considerando para el cálculo de la edad la fecha del reporte (31 de marzo de 2006).

Se pesquisaron 64 repeticiones de datos que fueron eliminadas del análisis. Para los 45.250 escolares seleccionados se generó la variable índice de masa corporal (IMC) que significó perder 12.015 observaciones por no contar todos con peso o talla para su cálculo. Entre las 33.235 observaciones de IMC se pesquisaron 721 valores incongruentes (IMC $<13$ para cualquiera edad y sexo) probablemente por error de digitación en la base original, que también fueron excluidos del análisis (Figura 1).

Para evaluar el efecto de la pérdida de las 12.736 observaciones comparamos las variables edad, género, comuna e índice de prioridad social entre la muestra excluyendo repeticiones $(\mathrm{N}=45.250)$ y la muestra analizada $(\mathrm{N}=32.514)$.
Para estimar las prevalencias de sobrepeso y obesidad se utilizaron los criterios del Comité de Expertos en Obesidad Infantil de los Estados Unidos de Norteamérica (EE.UU.)de 1998 con la referencia de IMC del CDC y la definición propuesta por la IOTF en 1997 utilizando como referente el estudio de Cole ${ }^{27-30}$. Las tablas con la referencia CDC de IMC en formato Excel para percentiles y $z$-scores se convirtieron a formato STATA y se fusionaron con los datos JUNAEB, homologando las variables edad y sexo. Los datos fueron analizados mediante el programa estadístico STATA 10.0 (STATA Corp.)

La prevalencia de sobrepeso y obesidad se expresó como porcentaje en relación al total de sujetos y luego según sexo, categorías etarias, índice de prioridad social familiar y comuna. A pesar de tratarse de un estudio de corte transversal se evaluó el riesgo de sobrepeso y obesidad de acuerdo al sexo, edad, índice de prioridad y comuna mediante odds ratio (OR). Finalmente, se evaluó el riesgo de sobrepeso y obesidad considerando todas las variables mencionadas mediante regresión logística múltiple.

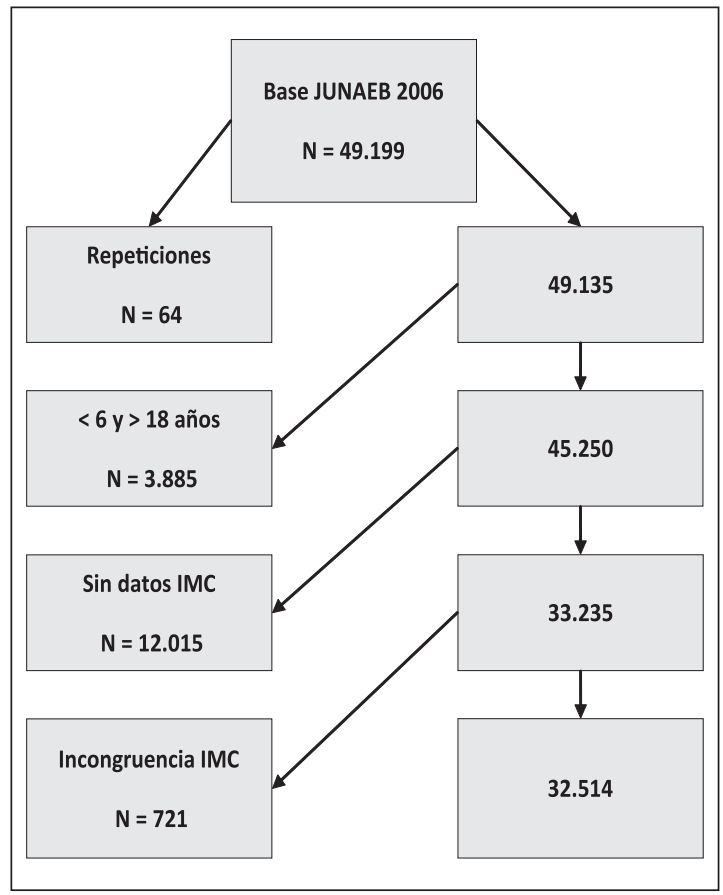

Figura 1. Obtención de la muestra de escolares para estimación de la prevalencia de obesidad y sobrepeso infanto- juvenil a partir de la base de datos JUNAEB Concepción, Coronel y Hualpén 2006. 
Sobrepeso y obesidad infanto-juvenil - N. Ulloa et al

\section{Resultados}

La edad promedio de la muestra analizada $(\mathrm{N}=32.514)$ fue $11,8 \pm$ 3,57 años (rango 5 a 17,8 años), con $47,55 \%$ de varones (IC 95\% 47-48). La proporción de familias socialmente vulnerables fue $65,3 \%$ en la Comuna de Concepción, 65,7\% en Hualpén y 78,77\% en Coronel. La edad promedio de la muestra original excluyendo repeticiones $(\mathrm{N}=45.250)$ fue 12,4 $\pm 4,32$ años (rango 5 a 17,8), con $47,93 \%$ de varones (IC 95\% 47,4-48,4). La proporción de familias socialmente vulnerables fue $66,1 \%$ en la Comuna de Concepción, 66,58\% en Hualpén y 79,46\% en Coronel. Al comparar estos datos, solamente la edad promedio de las muestras presentó una diferencia significativa $(\mathrm{p}<0,0001)$.

\section{Sobrepeso y obesidad según criterio CDC}

Las prevalencias de sobrepeso y obesidad de acuerdo al criterio percentilar del CDC fueron 11,19\% y $6,54 \%$ respectivamente. En la Tabla 1 se presentan la distribución de sobrepeso y obesidad según sexo, siendo el sobrepeso más frecuente en el sexo femenino y la obesidad en el masculino. En las Figuras 2 y 3 se puede apreciar el comportamiento de la prevalencia del sobrepeso y obesidad según edad, para cada sexo. Se observa una mayor frecuencia de sobrepeso y obesidad en el grupo etario entre $<8$ años, independientemente del sexo. Al contemplar las curvas de prevalencia de sobrepeso y obesidad versus edad, éstas adoptan la forma de $U$ con un nadir entre 8 y 12-13 años según sexo.

\section{Sobrepeso y obesidad según criterio IOTF}

Al realizar las estimaciones mediante el criterio IOTF se encontró que la prevalencia de sobrepeso fue
Tabla 1. Prevalencia de sobrepeso y obesidad en niños entre 6 y 18 años de las comunas de Concepción, Coronel y Hualpén según sexo (Criterio CDC). JUNAEB 2006

\begin{tabular}{|lccc|}
\hline Estado nutricional & $\begin{array}{c}\text { Varones } \\
\mathbf{n = 1 5 . 4 6 1}\end{array}$ & $\begin{array}{c}\text { Mujeres } \\
\mathbf{n = 1 7 . 0 5 3}\end{array}$ & \\
Categoría & $\mathbf{n ~ ( \% )}$ & $\mathbf{n ~ ( \% )}$ & Valor $\mathbf{p}$ \\
(Percentil) & $\mathbf{I C ~ 9 5 \%}$ & $\mathbf{I C} \mathbf{9 5 \%}$ & \\
\hline Sobrepeso (p85-95) & $1.637(10,6)$ & $2.002(11,74)$ & 0,0011 \\
& $10,1-11,08$ & $11,1-12,2$ & \\
Obeso (> p95) & $1.115(7,2)$ & $1.010(5,92)$ & $<0,0001$ \\
& $6,79-7,6$ & $5,55-6,27$ & \\
\hline
\end{tabular}

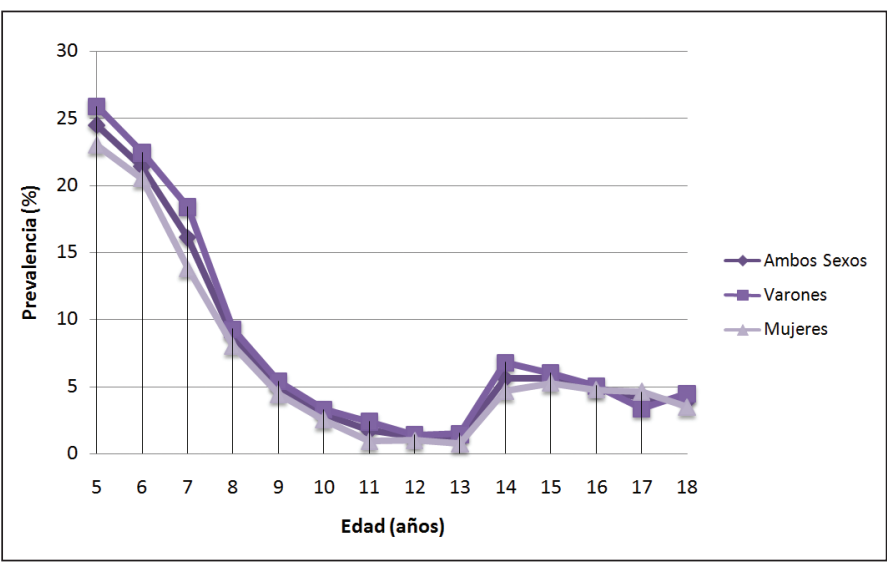

Figura 2. Prevalencia de obesidad según edad y sexo, de acuerdo al criterio CDC. JUNAEB 2006.

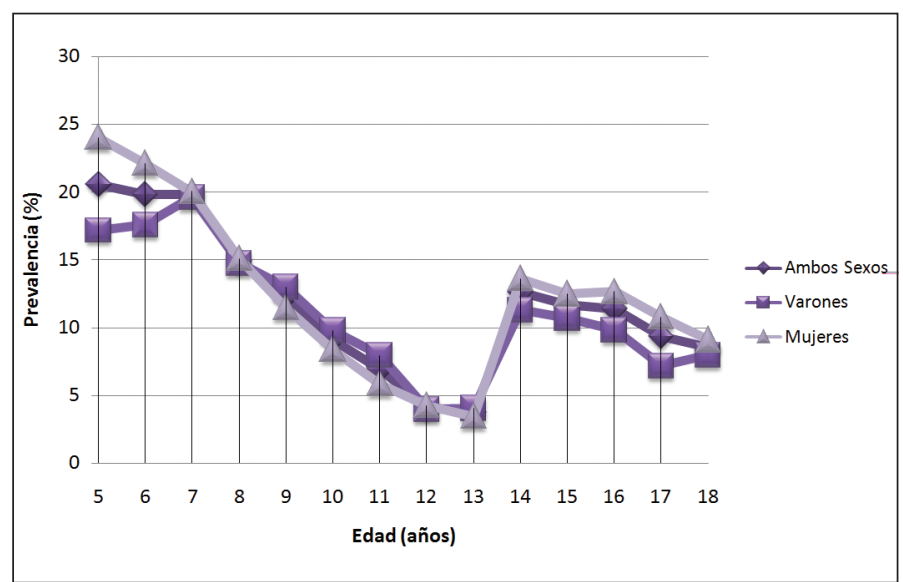

Figura 3. Prevalencia de sobrepeso según edad y sexo, de acuerdo al criterio CDC. JUNAEB 2006. 
$13,19 \%$ y la de obesidad $4,03 \%$. En la Tabla 2 se puede apreciar la prevalencia de sobrepeso y obesidad por sexo, destacando la mayor frecuencia de sobrepeso y obesidad en el sexo femenino. Este hallazgo contrasta con la mayor prevalencia de obesidad observada en varones con el criterio CDC. En la Figura 4 se puede observar que la variación de la prevalencia de sobrepeso y obesidad con la edad también asume la forma de $\mathrm{U}$ con el criterio IOTF.

La prevalencia de sobrepeso y obesidad según el criterio IOTF fueron significativamente diferentes a las obtenida por el criterio CDC (Tabla 3).

\section{Análisis de factores \\ de riesgo}

Al modelar mediante regresión logística la asociación entre prevalencia de sobrepeso y obesidad en conjunto con las variables sexo, edad (dicotomizada en menores y mayores de 8 años), comuna e índice de prioridad social, encontramos que tener menos de 8 años de edad (OR 3,96; IC 95\% 3,71- 4,21; p >0,0001) y residir en la comuna de Hualpén en relación a Concepción y Coronel(OR 1,16; IC 95\% 1,07-1,25; p = 0,0001) eran factores de riesgo para sobrepeso y obesidad.

Al modelar sólo prevalencia de obesidad como variable dependiente, encontramos mayor riesgo de esta condición en menores de 8 años (OR 5,66; IC 95\% 5,2-6,2; valor $\mathrm{p}<$ $0,0001)$, en el sexo masculino respecto al femenino (OR 1,24; IC 95\% 1,13$1,36 ; \mathrm{p}<0,0001)$ y en los residentes de Hualpén respecto a las otras comunas (OR 1,40; IC 95\% 1,3-1,6; p = 0,001).

$\mathrm{Al}$ construir un modelo predictivo del riesgo de obesidad mediante regresión logística múltiple se aprecia que la mayor parte del efecto se atribuiría a la edad dicotomizada en $<y>8$ años.
Tabla 2. Prevalencia de sobrepeso y obesidad en niños entre 6 y 18 años de las comunas de Concepción, Coronel y Hualpén según sexo (Criterio IOTF). JUNAEB 2006

\begin{tabular}{|c|c|c|c|}
\hline Estado nutricional & $\begin{array}{c}\text { Varones } \\
n=15.461\end{array}$ & $\begin{array}{c}\text { Mujeres } \\
n=17.053\end{array}$ & \\
\hline Categorías (IMC) & $\begin{array}{l}\text { n }(\%) \\
\text { IC } 95 \%\end{array}$ & $\begin{array}{c}\text { n }(\%) \\
\text { IC } 95 \%\end{array}$ & Valor $\mathbf{p}$ \\
\hline Sobrepeso (25-30) & $\begin{array}{c}1.780(11,51) \\
11,0-12,0\end{array}$ & $\begin{array}{c}2.490(14,6) \\
14,0-15,1\end{array}$ & $<0,0001$ \\
\hline Obesidad (> 30) & $\begin{array}{l}567(3,68) \\
3,38-3,97\end{array}$ & $\begin{array}{l}764(4,48) \\
4,16-4,79\end{array}$ & 0,0003 \\
\hline
\end{tabular}

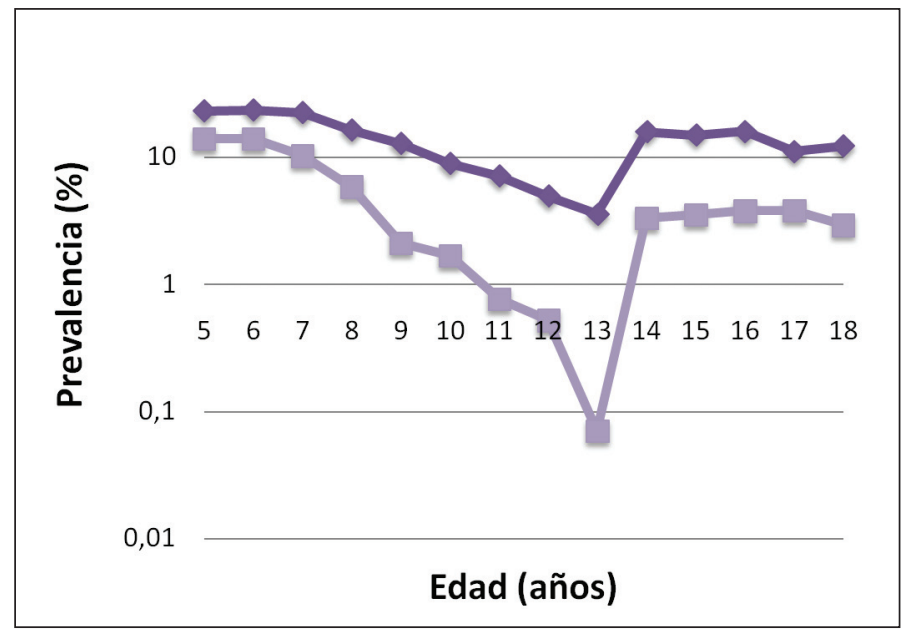

Figura 4. Prevalencia de obesidad de acuerdo al criterio IOTF. JUNAEB 2006.

Tabla 3. Prevalencia de sobrepeso y obesidad definidos por 2 criterios diagnósticos en niños entre 6 y 18 años de las comunas de Concepción, Coronel y Hualpén. JUNAEB 2006

\begin{tabular}{|lrrrr|}
\hline Estado nutricional & \multicolumn{2}{c}{$\begin{array}{c}\text { Varones } \\
\mathbf{n}=\mathbf{1 5 . 4 6 1}\end{array}$} & \multicolumn{2}{c|}{$\begin{array}{c}\text { Mujeres } \\
\mathbf{n}=\mathbf{1 7 . 0 5 3}\end{array}$} \\
& \multicolumn{2}{c}{ Criterio } & \multicolumn{2}{c|}{ Criterio } \\
& CDC & IOTF & CDC & IOTF \\
\hline Sobrepeso (\%) & 10,50 & $11,64^{*}$ & 11,74 & $14,60^{\&}$ \\
Obeso (\%) & 7,22 & $3,54^{* *}$ & 5,92 & $4,47^{8 \%}$ \\
\hline
\end{tabular}

${ }^{*} p=0,0014 .{ }^{* *} p<0,0001 .{ }^{*} p<0,0001 .{ }^{\&} \mathrm{p} p<0,0001$. 


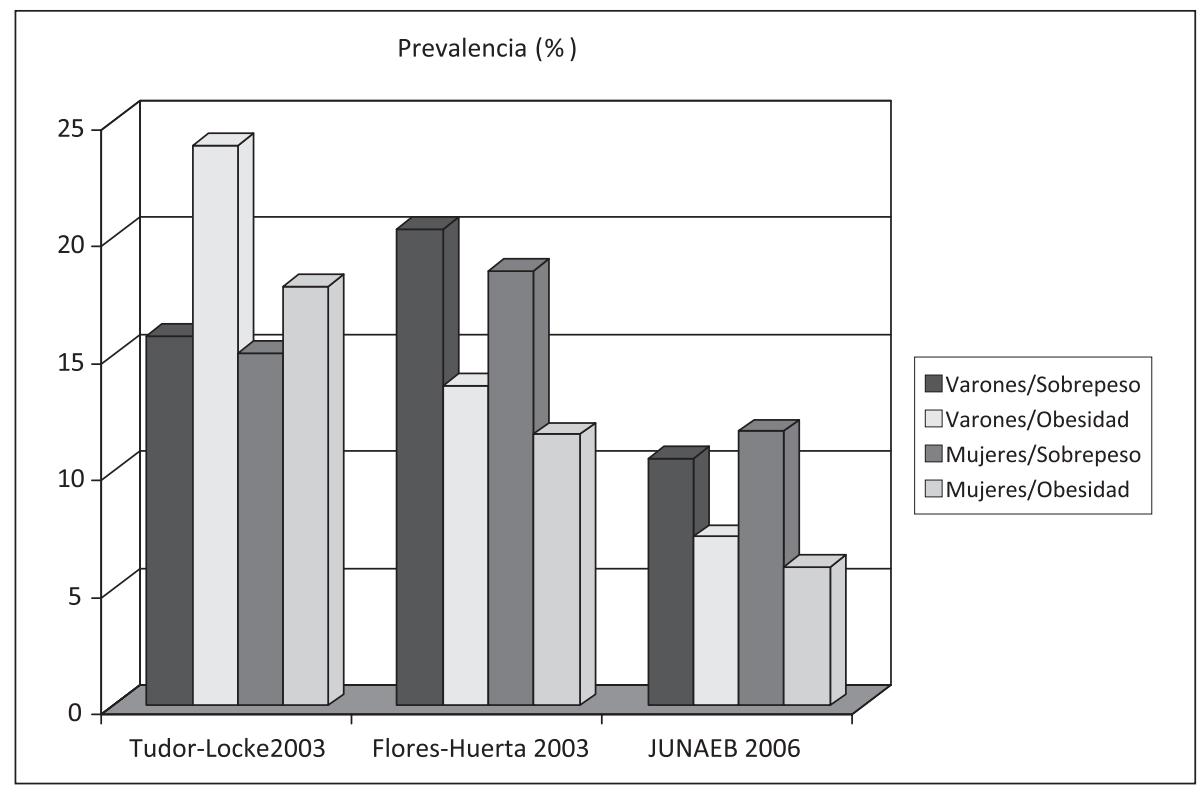

Figura 5. Prevalencia de sobrepeso y obesidad según sexo. Criterio CDC.

\section{Discusión}

Al comparar nuestros resultados con los obtenidos en estudios que utilizaron el criterio CDC a nivel nacional, encontramos mayor prevalencia de sobrepeso y obesidad en niños de 6 años que la reportada por Kain y colaboradores ${ }^{23}$. Este hecho no es sorprendente, considerando el aumento en la prevalencia observado en estudios de cortes transversales sucesivos y que nuestros datos fueron obtenidos 6 años después de los de Kain. Al comparar nuestros resultados con estudios internacionales encontramos menor prevalencia de obesidad y sobrepeso que 2 de ellos realizados mediante el criterio CDC en EE.UU. ${ }^{21}$ y México ${ }^{22}$, en grupos etarios semejantes y con datos obtenidos el año 2003 (Figura 5).

$\mathrm{Al}$ estimar la prevalencia de sobrepeso y obesidad mediante el criterio IOTF, nuestro estudio reportó una tasa mayor que la encontrada por Kain. Aunque el estudio GHSH también utilizó el criterio IOTF $^{24}$, no comparamos nuestros resultados con los de éste porque la forma en que obtuvieron el IMC (auto-reporte) subestima la prevalencia de sobrepeso y obesidad.

$\mathrm{Al}$ restringir la revisión de la literatura internacional a estudios que utilizaron el criterio IOTF, la mayor frecuencia de sobrepeso infanto-juvenil la tiene un estudio realizado en Grecia seguida de estudios de España, Alemania, Irlanda y Sue$\mathrm{cia}^{2-17,19}$. La mayor prevalencia de obesidad infantojuvenil la reportó un estudio de EE.UU. ${ }^{21}$. Otros estudios con prevalencias de obesidad superior al 10\% se realizaron en México, Kuwait, Grecia y España ${ }^{4,5,9,18,22}$.

En las Figuras 6 y 7 se comparan las prevalencias de sobrepeso en estudiantes menores y mayores de 12 años de nuestra muestra de población con las de otros estudios que utilizaron el criterio IOTF. En las Figuras 8 y 9 se comparan las prevalencias de obesidad. Se modificó la dicotomización de la variable edad ( 8 a 12 años) para permitir la comparación de datos. Al analizar las figuras se aprecia que en el grupo etario menor de 12 años de nuestra muestra tiene prevalencias de sobrepeso y obesidad relativamente más importantes que las que reportamos en mayores de 12 años.

Una de las variables que aumenta el riesgo de sobrepeso y obesidad es la condición socioeconómica familiar ${ }^{31-34}$. En el presente estudio, la falta de asociación entre prevalencia de sobrepeso y obesidad con el índice de prioridad social se puede atribuir a que en nuestra muestra las categoría sin o con grados leves de vulnerabilidad social estuvieron sub-representadas.

El resultado más relevante de nuestro estudio es que el sub-grupo de niños menores de 8 años tiene la mayor prevalencia de sobrepeso y obesidad. Este 


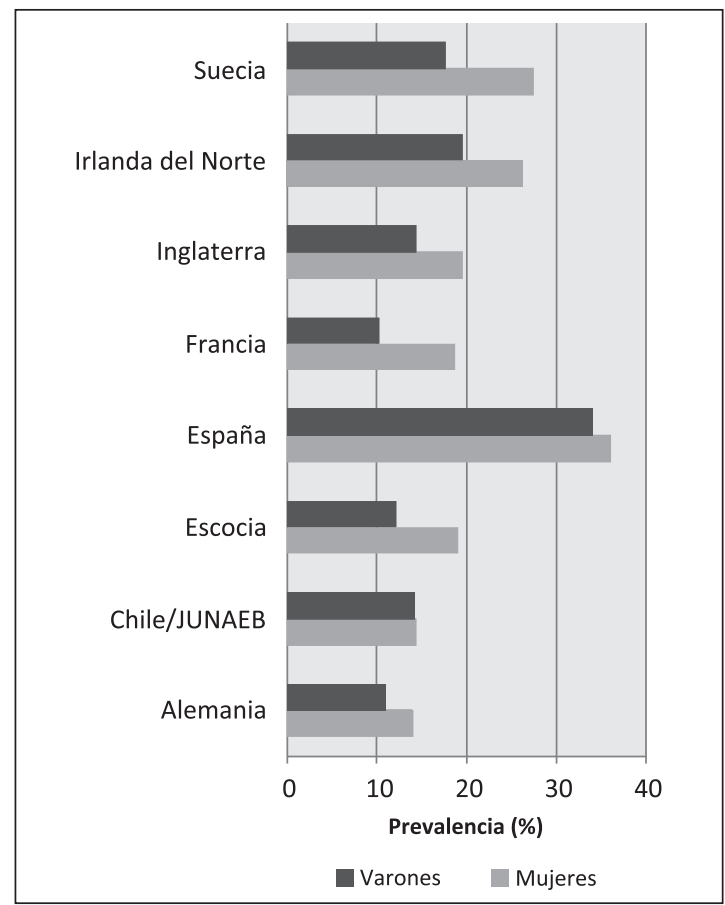

Figura 6. Prevalencia de sobrepeso en estudiantes $<12$ años según sexo. Criterio IOTF.

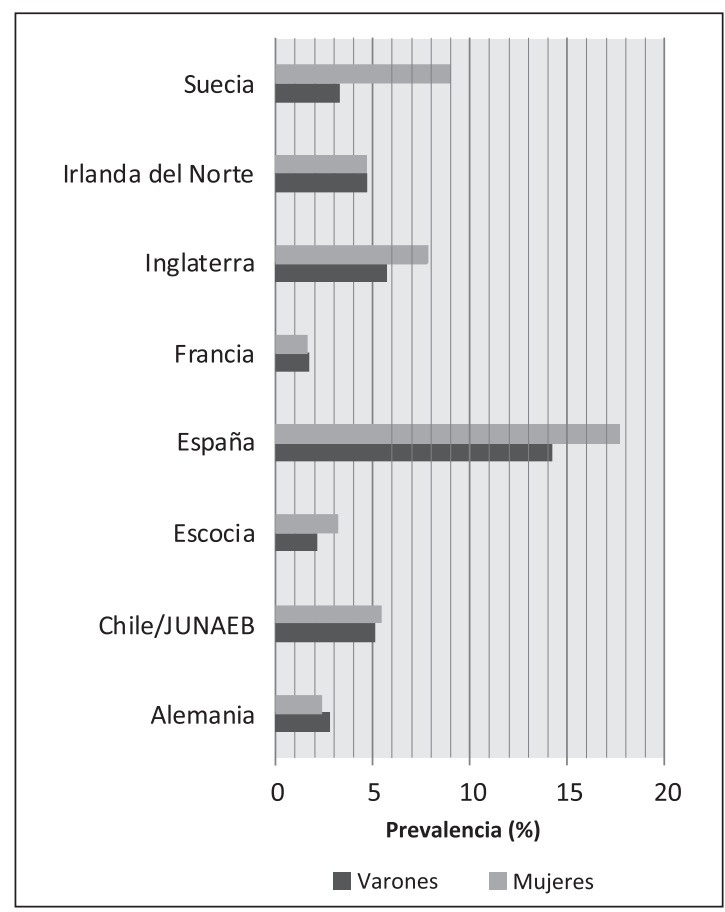

Figura 8. Prevalencia de obesidad en estudiantes $<12$ años según sexo. Criterio IOTF.

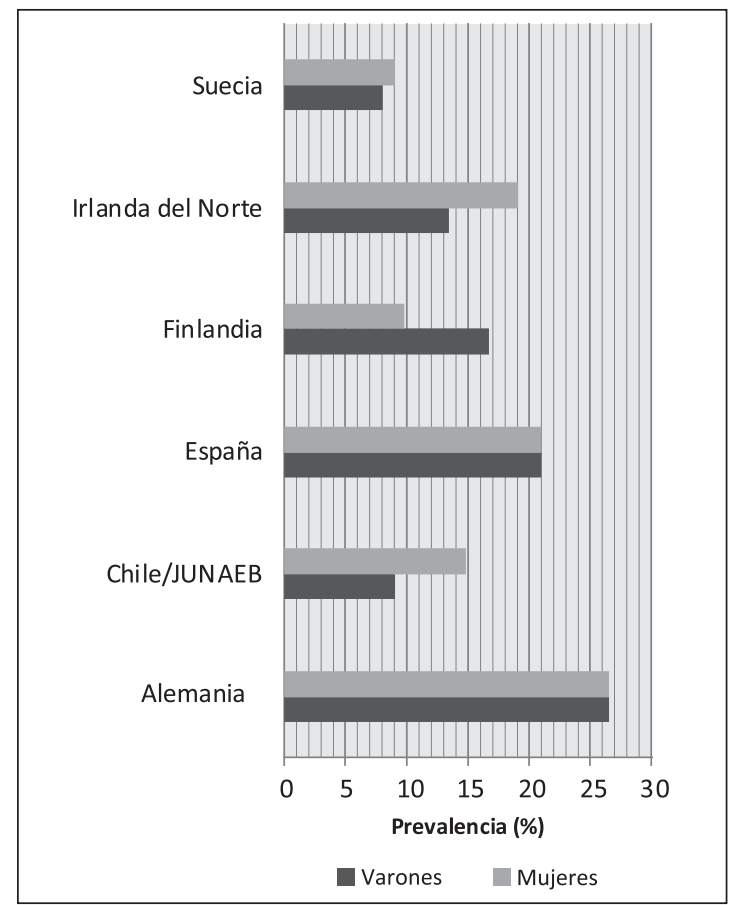

Figura 7. Prevalencia de sobrepeso en estudiantes $>12$ años según sexo. Criterio IOTF.

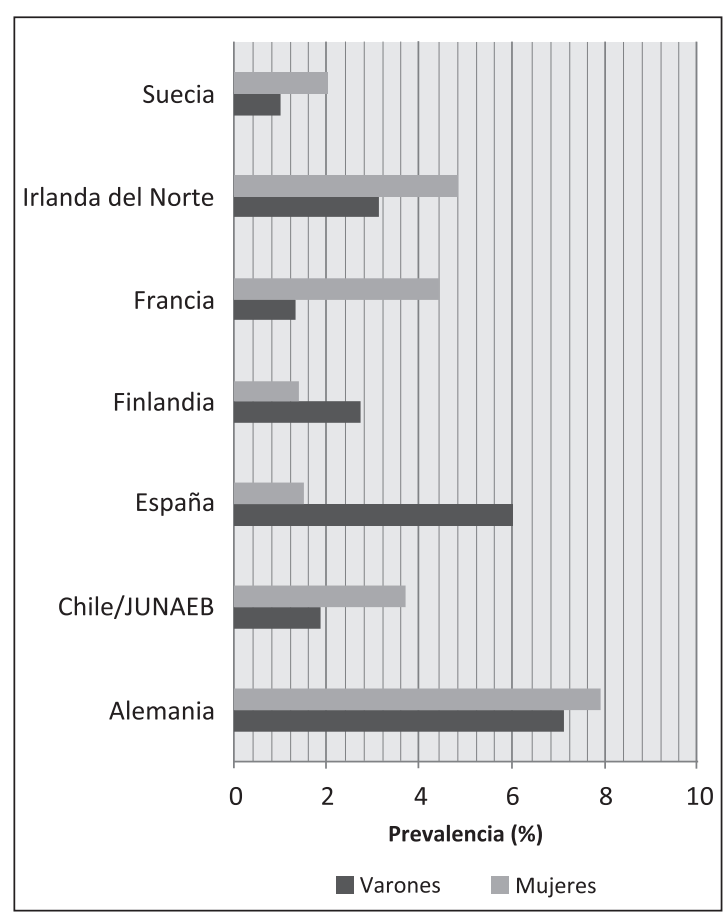

Figura 9. Prevalencia de obesidad en estudiantes $>12$ años según sexo. Criterio IOTF. 
Sobrepeso y obesidad infanto-juvenil - N. Ulloa et al

hallazgo es proporcionalmente más importante en nuestra muestra que en estudios internacionales que utilizaron el criterio IOTF. La forma de U de la curva de prevalencia de sobrepeso y obesidad versus edad se podría interpretar como un fenómeno ligado al crecimiento, sin embargo, el nuestro es un estudio de corte transversal y no una cohorte, por lo que también puede representar diferencias en el comportamiento nutricional entre diferentes generaciones. Este hecho fue reportado por Muzzo y cols ${ }^{35}$, quienes en un análisis de estudios de corte transversal consecutivos encontraron un incremento más pronunciado en la proporción de sobrepeso y obesidad en sujetos pre-puberales (Criterio CDC).

A pesar que la base de datos JUNAEB 2006 evidencia las incongruencias y omisiones propias de los registros poblacionales y que no podemos excluir algún grado de sesgo de selección relacionado con la exclusión de los sujetos sin registro de antropometría, nuestros resultados nos permiten sugerir que la prevalencia del sobrepeso y obesidad infanto-juvenil ha aumentado con el tiempo y que los niños menores de 8 años representan un subgrupo de alto riesgo de estas enfermedades nutricionales. En consecuencia, la población infantil menor de 8 años de edad debiera ser el objetivo principal de los programas de intervención que se elaboren en el futuro.

Agradecimientos: Queremos agradecer la valiosa cooperación de la Sra Cristina Miranda del Centro Saval Temuco en la obtención de las referencias bibliográficas para el estudio.

\section{Referencias}

1. Encuesta Nacional de Salud 2003: http://epi.minsal.cl// Epi/Html/Invest/ENS/InformeFinalENS.

2. Frye C, Heinrich J. Trends and predictors of overweight and obesity in East German children. Int J Obes Relat Metab Disord 2003; 27: 963-9

3. Chinn S, Rona RJ. Prevalence and trends in overweight and obesity in three cross sectional studies. BMJ 2001; 322: 24-6.

4. Moreno LA, Sarria A, Popkin BM. The nutrition transition in Spain: a European Mediterranean country. Eur J Clin Nutr 2002; 56: 1-12.

5. Briz FJ, Cos AI, Amate AM. Prevalencia de obesidad infantil en Ceuta. Estudio PONCE 2005. Nutr Hosp 2007;
22: 471-7.

6. Kautiainen S, Rimpëlla A, Vikat SM. Secular trends in overweight and obesity among Finnish adolescents in 1977-1999. Int J Obes Relat Metab Disord 2002; 26 : 544-52.

7. Heude B, Lafay L, Borys JM, Thibult N, Lommez A, Romon $\mathrm{M}$, et al. Time trend in height, weight and obesity prevalence in school children from Northen France. Diabetes Metabolism 2003; 29: 355-40.

8. Romon M, Duhamel A, Collinet N, Weill J. Influence of social class on time trends in BMI distribution in 5 year old French children from 1989-1999. Int J Obes Relat Metab Disord 2005; 29: 54-9.

9. Magkos F, Manios Y, Christakis G, Kafatos AG. Secular trends in cardiovascular risk factors among school-aged boys from Creta, Greece. Eur J Nutr 2005; 59: 1-7.

10. Wit JM, Verloove-Vanhorick SP. Body index measurements in 1996-7 compared 1980. Arch Dis Child 2000; 82: $107-12$.

11. Lobstein TJ, James WP, Cole TJ. Increasing levels of excess weight among children in England. Int J Obes Relat Metab Disord 2003; 27: 1136-8.

12. Watkins DC, Murray LJ, Mccarron P, Boreham CAG, Cran GW, Young IS, et al. Ten-year trends for fatness in Northern Irish adolescents: the Young Hearts Projectsrepeat cross sectional study. Int J Obes Relat Metab Disord 2005; 29: 579-85.

13. Whelton H, Harrington J, Crowley E, Kelleher V, Cronin M, Perry IJ. Prevalence of overweight and obesity on the island of Ireland: Results from the North South Survey of Children's Height, Weight and Body Mass Index, 2002. BMC Public Health 2007; 7: 187-95.

14. Petersen S, Brulin C, Bergstrom E. Increasing prevalence of overweight in young schoolchildren in Umea, Sweden, from 1986 to 2001. Acta Paediatrica 2003; 92: 848-53.

15. Holmbäck U, Fridman J, Gustafsson J, Proos L, Sundelin C, Forslund A. Overweight more prevalent among children than among adolescents. Acta Paediatrica 2006; 96: 577-81.

16. Magarey AM, Daniels LA, Boulton TJ. Prevalence of overweight and obesity in Australian children and adolescents: reassessment of 1985 and 1995 data against new standard international definitions. Med J Aust 2001; 174: 561-4.

17. Matsushita Y, Yoshiike N, Kaneda F, Yoshita K, Takimoto H. Trends in childhood obesity in Japan over the last 25 years from the national nutrition survey. Obes Res 2004; 12: 205-14.

18. Jackson Rt, Rashed M, Al-Hamad N, Hwalla N, AlSomaie M. Comparison of BMI-for-age in adolescents 
Sobrepeso y obesidad infanto-juvenil - N. Ulloa et al

girls in 3 countries of the Eastern Mediterranean Region. La Revue de Santé de la Mediterranée orientale 2007; 13: 430-9.

19. Tremblay MS, Katzmarzyk PT, Willms JD. Temporal trends in overweight and obesity in Canada, 1981-1996. Int J Obes Relat Metab Disord 2002; 26: 538-43.

20. Willms JD, Tremblay MS, Katzmarzyk PT. Geographic and demographic variations in the prevalence of overweight Canadian children. Obes Res 2003; 11: 668-73.

21. Tudor-Locke C, Kronenfeld JJ, Kim SS, Benin M, Kuby M. A geographical comparison of prevalence of overweight school-aged children: The Nacional Survey of Children's Health 2003. Pediatrics 2007; 120: e1043-50.

22. Flores-Huerta S, Acosta-Cazares B, Gutiérrez-Trujillo G. Prevalencia de bajo peso, sobrepeso, obesidad general y central. Rev Med Inst Mex Seguro Soc 2006; 44: s55-s62.

23. Kain J, Uauy R, Vio F, Albala C. Trends in overweight and obesity prevalence in Chilean children: comparison of three definitions. Eur J Clin Nutr 2002; 56: 200-4.

24. Global School-Based Student Health Survey (2004-2005) Chile: http://epi.minsal.cl/epi/html/frames/frame3.htm.

25. Tokmakidis SP, Christodoulos Ad, Mantzouranis NI. Validity of self-reported anthropometric values used to assess body mass index and estimate obesity in Greek school children. J Ado Health 2007; 40: 305-10.

26. Fergadiot A. Indice de prioridad social Región Metropolitana 2007: actualización http://www.serplacrm.cl/ publicaciones/estudios/INDICE_DE_PRIORIDAD_SOCIAL_2007.pdf.

27. Barlow S, Dietz W. Obesity evaluation and treatment: expert committee recommendations. Pediatrics. 1998; 102: E29-E36.

28. CDC/NCHS (2000) CDC growth charts: United States. http//www.cdc.gov/growthcharts.Posted May 30, 2000 in the Internet.

29. Troiano R, Flegal K. Overweight prevalence among youth in the US: why so many different numbers. Int J Obes Relat Metab Disord 1999; 23 (Suppl 2): S22-7.

30. Cole T, Bellizzi M, Flegal K, Dietz W. Establishing standard definition for child overweight and obesity worldwide: international survey. BMJ 2000; 320: 1-6.

31. Lioret S, Touvier M, Dubuisson C, Dufour A, CalamassiTran G, Lafay L, et al. Trends in child overweight rates and energy intake in France from 1999 to 2007: relationships with socioeconomic status. Obesity (Silver Spring) 2009; 17: 1092-100.

32. Amigo H, Bustos P, Erazo M, Cumsille P, Silva C. Factores determinantes del exceso de peso en escolares: Un estudio multinivel. Rev Med Chile 2007; 135: 1510-8.

33. Olivares S, Bustos N, Lera L, Zelada ME. Estado nutricional, consumo de alimentos y actividad física en escolares mujeres de diferente nivel socioeconómico de Santiago de Chile. Rev Med Chile 2007; 135: 71-8.

34. Shrewsbury V, Wardle J. Socioeconomic status and adiposity in childhood: a systematic review of crosssectional studies 1990-2005. Obesity (Silver Spring) 2008; 16: 275-84.

35. Muzzo S, Burrows R, Corder J, Ramírez I. Trends in nutritional status and stature among school-age children in Chile. Nutrition 2004; 20: 867-72. 